\title{
Unsteady Effects in Droplet Evaporation and Combustion
}

\author{
A. CRESPO and A. LIÑAN \\ Instituto Nacional de Técnica Aeroespacial "Esteban Terradas", Madrid, Spain
}

\section{INTRODUCTION}

The evaporation or simultaneous evaporation and combustion of a fuel droplet is frequently treated by using quasisteady theories (see the review papers by Williams (1973) and Rosner (1972)). This assumption is based on the facts that the droplet is burning or vaporizing in a steady environment and that the liquid density is much higher than the gas density (Williams, 1959). The purpose of this work is to make a perturbation analysis in which, by considering the ratio of the densities of gas and liquid as a small parameter, the asymptotic nature of the quasisteady solution is shown. Corrections to the "quasisteady" $(Q S)$ theory are found to be of the order of the square root of the ratio of the densities. It is also found that the solution is "nonsteady" $(N S)$ at distances from the droplet of the order of the droplet radius divided by the square root of the ratio of the densities.

A situation in which $N S$ effects are important is when the pressure and temperature at the droplet surface approach the critical pressure and temperature of the fuel (Rosner and Chang, 1973). This may happen in liquid propellant rocket motors and in diesel engines (Wieber, 1963; Rosner, 1967; Manrique and Borman, 1969). For high temperature and pressure the non-ideal gas effects and the dissolution of the ambient fluid in the liquid phase have to be taken into account (Manrique and Borman, 1969; Matlosz, Leipziger and Torda, 1972). Manrique and Borman used the $Q S$ approximation in their works; this may be justified when the ambient density is much smaller than the critical density of the fuel; in this case the $Q S$ results can be improved with an analysis similar to the one presented here. In our analysis, however, we will not take into account the realistic gas mixture properties and it will be assumed that the ambient gas is not dissolved in the droplet, and that the droplet temperature is the boiling temperature at ambient pressure. This last assumption is based on the fact that the heat of vaporization divided by the specific heat at constant pressure is large compared to the boiling temperature, and implies the existence of a heat-up period that precedes the vaporization period, during which there is no significant vaporization. This heat up period is not considered here. In this work we will mainly concentrate in calculating the perturbations introduced to the results of the $Q S$ analysis by $N S$ effects during the vaporization period.

A comparison will be made of our perturbation theory results and the numerical results of Duda and Vrentas (1971) for the problem of sphere dissolution, recently shown by Rosner and Chang (1973) to be applicable to the prediction of fuel droplet lifetimes when $Q S$-approximations fail.

The problem of droplet evaporation with combustion is reduced to the pure evaporation case, for Lewis number equal to one, by using the thin flame assumption. It is found that when the stoichiometric ratio is not large, the flame position is correctly predicted by the $Q S$ theory, and corrections to this result are of the order of the square root of the ratio of the densities. However, in many practical cases the stoichiometric ratio is large and the flame has to go far from the droplet so that fuel and oxidizer will reach it in stoichiometric ratio. When the flame is in the $N S$ region, the $Q S$ theory will not give correctly its position even in first 
approximation. The analysis presented here explains the fact (experimentally confirmed) that in many cases the $Q S$ analysis gives valid results regarding burning rates but not regarding flame position (Nuruzzaman and Beer, 1971; Krier and Wronkiewic, 1972; Rush and Krier, 1973; Isoda and Kumagai, 1958; Kumagai, Sakai, Okajima, 1971). An earlier attempt to solve this contradiction has been made by Chervinsky (1969), however, the mathematical approximations he uses do not appear to be justified, and his theory does not lead to useful results for the $N S$-droplet lifetime. Kotake and Okazaki (1969) obtained numerical solutions of the $N S$ equations of droplet combustion that show a qualitative agreement with the above-mentioned experimental results.

The results obtained here show that the time elapsed until the flame radius vanishes (extinction time) is longer than the vaporization time. When the stoichiometric ratio is not large, these two times are approximately the same, however, for sufficiently large values of that ratio the extinction time may even become much larger than the vaporization time. In this last limiting case our results coincide with those obtained by Spalding (1959) who replaces the droplet by an instantaneous source of vapor.

The problem of supercritical droplet combustion has been shown by Tarifa, Crespo, and Fraga (1972), using a gas pocket model, to be similar in many aspects to the subcritical case if the initial pocket temperature is much smaller than the ambient temperature. However, for supercritical droplet combustion the $N S$ effects are more important than in the case analyzed here, because the ratio of the two temperatures mentioned earlier is usually not so small as the ratio of the gas and liquid densities. These $N S$ effects have been considered by Tarifa and Crespo (1972) in an analysis similar to the one presented here.

\section{MODEL AND ASSUMPTIONS}

The configuration to be used is shown in Figure 1 . An isolated fuel droplet is enveloped by a very thin flame where fuel and oxidizer react irreversibly at an infinetely fast rate. The fuel concentration is zero outside the flame, and the oxidizer concentration is zero inside the flame. Both concentrations vanish at the flame. For the pure vaporization case the fuel concentration at infinity may be different from zero.

It will be assumed that the fluid properties

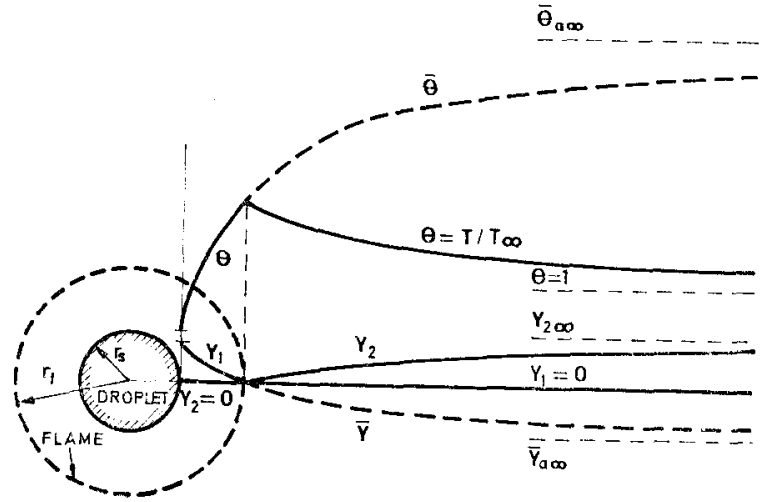

FIG. 1. Schematic showing temperature and concentration distributions, for droplet evaporation with thin flame combustion.

initially are uniform and at infinity are uniform and constant, and that there are no forced convection effects. These assumptions imply that there is spherical symmetry. Tarifa and Crespo (1972) show that forced convection effects are negligible compared to $N S$ effects when the Reynolds number corresponding to the external velocity is small compared to the square root of the ratio of gas and liquid density.

We consider conditions at the vapor/liquid interface to be well below the critical conditions of the fuel, so that we can assume that there is no dissolution of ambient gas in the droplet. Since, under these conditions, the heat of vaporization is large, the droplet temperature is very close to the boiling temperature of the fuel, except for a heat-up period which is not analyzed in this work; the droplet is here assumed to be always at the boiling temperature.

The gas is assumed to be a perfect gas mixture with a constant value of the mean molecular mass and of the specific heat, $c_{p}$. Since we will neglect the effects of the small spatial variations of pressure in the equation of state, the product of the density times the temperature will be constant

$$
\rho T=\rho_{\infty} T_{\infty}
$$

Only a diffusion coefficient has been considered, which, for simplicity in the presentation, has been assumed to be inversely proportional to the density

$$
\rho D=\rho_{\infty} D_{\infty}
$$

and equal to the thermal diffusitivity of the mixture

$$
\rho c_{p} D / \lambda=1
$$

In the following equations non-dimensional variables will be used. The temperature and the 
density have been made non-dimensional with their corresponding values at infinity:

$$
\theta=T / T_{\infty} \quad \bar{\rho}=\rho / \rho_{\infty}
$$

The radial distance and the instantaneous droplet radius are made non-dimensional with the initial droplet radius:

$$
x=r / r_{s o} \quad x_{s}=r_{s} / r_{s o}
$$

The non-dimensional time is:

$$
\tau=\varepsilon D_{\infty} t / r_{s o}
$$

where $\varepsilon$ is the ratio of the ambient gas and liquid densities

$$
\varepsilon=\rho_{\infty} / \rho_{l}
$$

This non-dimensional form of the time has been chosen so as to make the non-dimensional vaporization time of order unity.

The velocity is made non-dimensional with a characteristic gas velocity obtained by dividing the initial droplet radius by the diffusion time

$$
\bar{V}=V\left(r_{s o}^{2} / D_{\infty}\right) / r_{s o}
$$

In the following the suscript $s$ also means gas conditions at the droplet surface.

\section{DROPLET VAPORIZATION}

Although the theory presented here could be applied to the case of vaporization with combustion, the method is better illustrated with this more simple case. Nevertheless, the main results of the combustion case will be exposed at the end.

The equations to be considered are, in nondimensional form, as follows:

Mass conservation

$$
\varepsilon \frac{\partial \bar{\rho}}{\partial \tau}+\frac{1}{x^{2}} \frac{\partial}{\partial x}\left(x^{2} \bar{\nabla} \bar{\rho}\right)=0
$$

Species conservation for the mass fraction, $Y_{1}$, of the fuel vapor

$$
\mathscr{L}\left(Y_{1}\right)=0
$$

Energy conservation

$$
\mathscr{L}(\theta)=0
$$

where

$$
\mathscr{L}=\varepsilon \bar{\rho} \frac{\partial}{\partial \tau}+\bar{\rho} \bar{V} \frac{\partial}{\partial x}-\frac{1}{x^{2}} \frac{\partial}{\partial x}\left(x^{2} \frac{\partial}{\partial x}\right)
$$

Equation of state:

$$
\bar{\rho} \theta=1
$$

The boundary conditions are:

Mass conservation at the droplet surface

$$
\frac{d x_{s}}{d \tau}=-\frac{1}{\theta_{s}}\left(\bar{V}_{s}-\varepsilon \frac{d x_{s}}{d \tau}\right)
$$

Mass conservation for evaporation fuel

$$
\left(1-Y_{1 s}\right)\left(\bar{V}_{s}-\varepsilon \frac{d x_{s}}{d \tau}\right)+\left.\theta_{s} \frac{\partial Y_{1}}{\partial x}\right|_{s}=0
$$

Energy conservation

$$
\left.\frac{\partial \theta}{\partial x}\right|_{s}=\frac{1}{\theta_{s}}\left(\bar{V}_{s}-\varepsilon \frac{d x_{s}}{d \tau}\right) \frac{L}{c_{p} T_{\infty}}+\frac{x_{s}}{3} \frac{c_{p l}}{c_{p}} \frac{d \theta_{s}}{d \tau}
$$

Clapeyron equation, for constant heat of vaporization, $L$, per unit mass of fuel,

$$
Y_{1 s}=\exp \left[-\frac{L}{R_{1} T_{B}}\left(\frac{\theta_{B}-\theta_{s}}{\theta_{s}}\right)\right]
$$

where $R_{1}$ is the gas constant for the fuel vapor. If $L / R_{1} T_{B}$ is large, $Y_{1 s}$ will be very small until $\theta_{s}$ becomes almost equal to $\theta_{B}$. Thus, there will be a heat-up period during which the droplet temperature rises to the boiling temperature, followed by the vaporization period which we shall analyze here with the assumption

$$
\theta_{s}=\theta_{B}=T_{B} / T_{\infty}
$$

The conditions at infinity are:

$$
\begin{gathered}
x \rightarrow \infty: \theta=1 \quad Y_{1}=Y_{1 \infty} \\
\tau=0 \quad x>1: \theta=1 \quad Y_{1}=Y_{1 \infty}
\end{gathered}
$$

In these equations and boundary conditions $\varepsilon$ is a small parameter; if it is made equal to zero, the classical results of the $Q S$ theory of droplet vaporization are obtained.

\subsection{QS Solution}

The mass conservation equation (1) gives

$$
x^{2} \bar{V} / \theta=m(\tau) x_{s}
$$

where $m$ is an integration constant that, from equation (6), is given by

$$
m=x_{s} \bar{V}_{s} / \theta_{s}=-\frac{1}{2} d x_{s}^{2} / d \tau
$$

By integrating equation (3) with boundary conditions (8) and $\left(9^{\prime}\right)$

$$
\theta=\theta_{B}-\frac{L}{c_{p} T_{\infty}}+\frac{L}{c_{p} T_{\infty}} \exp \left[m\left(1-x_{s} / x\right)\right]
$$


This expression for large values of $x$ behaves like

$$
\theta=\theta_{B}-\frac{L}{c_{p} T_{\infty}}+\frac{L}{c_{p} T_{\infty}}\left(1-m x_{s} / x\right) \exp m
$$

showing that, far from the droplet, the temperature distribution is that corresponding to a heat source of strength $-m x_{s} \exp (m) L / c_{p} T_{\infty}$, without radial convective effects, in an atmosphere of temperature $\theta_{\infty a}$

$$
\theta_{\infty a}=\theta_{B}+\frac{L}{c_{p} T_{\infty}}(\exp m-1)
$$

If the boundary condition (10) is taken into account, $\theta_{\infty a}$ is equal to one, obtaining

$$
m=m_{0}=\ln \left\{1+\left(1-\theta_{B}\right) c_{p} T_{\infty} / L\right\}
$$

and from equation (13) and the initial condition $x_{s}=1$ at $\tau=0$

$$
x_{s}^{2}=x_{s o}^{2}=1-2 m_{0} \tau
$$

By integrating equation (3) with boundary conditions (10) and (7), the concentration field is obtained

$$
Y_{1}=1-\left(1-Y_{1 \infty}\right) \exp \left(-m x_{s} / x\right)
$$

The temperature and concentration fields are given by equations (15) and (19) with $m$ and $x_{s}$ given by equations (17) and (18). Notice that, far from the droplet, the concentration distribution corresponds to a mass source of strength $\left(1-Y_{1 \infty}\right) m_{0} x_{s o}$. When trying to improve this $Q S$ solution by using a regular expansion procedure, the next terms, of order $\varepsilon$, are found to diverge at infinity (Williams, 1959).

This difficulty is mathematically similar to that appearing when trying to find a second approximation to the slow viscous motion around a sphere (Whitehead's paradox), and the method used by Kaplun and Lagerstrom (1957) to solve this problem will be applied here. There is an "Outer Region" located at distances from the droplet of the order of

$$
x \sim 1 / \sqrt{\varepsilon}
$$

where the $N S$ and diffusion terms are of the same order in equations (2) and (3), and where the temperature and concentration differ from their values at infinity (10) by small terms, of order $\sqrt{\bar{\varepsilon}}$, which will be obtained below by linearization of equations (2) and (3). In an "Inner Region", located at distances from the droplet of the order of the droplet radius, the $Q S$ differential equations describe the temperature and concentration distributions, with errors of order $\varepsilon$, if the appropriate matching conditions with the outer expansion are used. Since the first approximation to the outer solution is given by conditions at infinity (10), the classical $Q S$ results presented above, with the condition $\theta_{a \infty}$ equal to one, correspond to the first approximation to the solution with errors of order $\sqrt{\varepsilon}$.

\subsection{Outer Solution}

In this region the appropriate variables are $\tau$, and

$$
X=x \sqrt{\varepsilon}
$$

and we use the expansion

$$
\begin{aligned}
\theta & =1+\sqrt{\varepsilon} \theta^{*}+0(\varepsilon) \\
Y_{1} & =Y_{1 \infty}+\sqrt{\varepsilon} Y_{1}^{*}+0(\varepsilon)
\end{aligned}
$$

where $\theta^{*}$ and $Y_{1}^{*}$ are functions of $X$ and $\tau$.

The continuity equation (1) will only be used to show that $\bar{V}$ is of order $\varepsilon$ in this region, and consequently the convective term is of order $\sqrt{\varepsilon}$ relative to the NS term in equations (2) and (3). By taking equations (20) and (21) into equation (3), the equation for the temperature perturbation becomes

$$
\frac{\partial \theta^{*}}{\partial \tau}=\frac{1}{x^{2}} \frac{\partial}{\partial x}\left(x^{2} \frac{\partial \theta^{*}}{\partial x}\right)
$$

A similar equation is obtained for the concentration. In order to match with the inner solution (15), the solution of (23), satisfying the initial condition $\theta^{*}=0$ (equation (11)), is written in the form

$$
\theta^{*}=\frac{1}{2 \sqrt{\pi}} \int_{0}^{\tau} g(\xi) \frac{e^{-X^{2} / 4(\tau-\xi)}}{(\tau-\xi)^{3 / 2}} d \xi
$$

that expresses that the droplet, when viewed from a large distance, behaves like a point heat source of varying intensity, $g(\tau)$. The same solution (24) is valid for the concentration, $Y_{1}^{*}$, with a different source intensity, $h(\tau)$.

At small values of $X$, and large values of $x$, given by $\tilde{x} \sim 1$ in an intermdeiate variable

where

$$
\tilde{x}=\eta x
$$

$$
\sqrt{\varepsilon} \ll \eta \ll 1
$$

the first two terms of the outer expansion (21) lead to

$$
\begin{aligned}
\theta=1 & +\frac{g\left(\tau_{1}\right) \eta}{\tilde{x}}-\frac{\sqrt{\varepsilon}}{\sqrt{\pi}} \\
& \times\left[\frac{g(0)}{\sqrt{\tau_{1}}}+\int_{0}^{\tau_{1}} \frac{g^{\prime}\left(\tau_{1}-\xi\right)}{\sqrt{\xi}} d \xi\right]+\sigma(\sqrt{\varepsilon})
\end{aligned}
$$


and a similar equation for $Y_{1}$ with $g$ replaced by $h$ and the first term in the right hand side replaced by $Y_{1 \infty}$. The matching conditions with the first approximation to the inner solution, or classical $Q S$ solution, give

$$
\begin{aligned}
& g(\tau)=-\frac{L}{c_{p} T_{\infty}} m_{0} \exp \left(m_{0}\right)\left(1-2 m_{0} \tau\right)^{1 / 2} \\
& h(\tau)=\left(1-Y_{1 \infty}\right) m_{0}\left(1-2 m_{0} \tau\right)^{1 / 2}
\end{aligned}
$$

\subsection{Inner Solution}

As it was indicated before, the inner solution is given in first approximation by equations (14) and (19), with $m$ and $x_{s}$ given by equations (17) and (18) which result from the matching condition with the first approximation of the outer solution

$$
\theta=1 \quad Y_{1}=Y_{1 \infty} \quad \text { at } x \rightarrow \infty
$$

To calculate corrections of order $\sqrt{\varepsilon}$ to this $Q S$ solution, the $Q S$ differential equations and boundary conditions at the droplet surface can still be used, however, the outer boundary condition (29) should be substituted by the matching conditions with the two-term outer expansion. Then,

$$
\begin{aligned}
\theta & =1+\sqrt{\varepsilon} \theta_{a}^{*}(\tau) \\
Y_{1} & =Y_{1 \infty}+\sqrt{\varepsilon} Y_{1 a}^{*}(\tau) \text { at } x \rightarrow \infty
\end{aligned}
$$

where $\sqrt{\varepsilon} \theta_{a}^{*}$ is the third term in the right hand side of equation (26), and $\sqrt{\varepsilon} Y_{1 a}^{*}$ is the corresponding term for $Y_{1}$.

Then, the first two terms of the inner expansion can be easily obtained from equation (14) with $m$ given by the linearized form of equation (16) with

$$
\theta_{\infty a}=1+\sqrt{\varepsilon} \theta_{a}
$$

and $x_{s}^{2}$ given by (13). Thus, we obtain

$$
\left(m-m_{0}\right) / \sqrt{\varepsilon}=m_{1}=\left(1-\theta_{B}+L / c_{p} T_{\infty}\right)^{-1} \theta_{a}^{*}
$$

and using equations (26) and (30)

$$
m_{1}=\frac{m_{0}^{3 / 2}}{\sqrt{\pi}}\left[\frac{1}{\sqrt{\tau m_{0}}}-\frac{1}{\sqrt{2}} \ln \frac{1+\sqrt{2 m_{0} \tau}}{1-\sqrt{2 m_{0} \tau}}\right]
$$

From equation (13)

$$
\begin{aligned}
X_{s}^{2}=1 & -2 m_{0} \tau-\left(2 \varepsilon m_{0} / \pi\right)^{1 / 2} \\
& \times\left[\sqrt{2 m_{0} \tau}+\frac{1-2 m_{0} \tau}{2} \ln \frac{1+\sqrt{2 m_{0} \tau}}{1-\sqrt{2 m_{0} \tau}}\right]
\end{aligned}
$$

The proportionality of the heat and mass sources, equations (27) and (28), allows us to write

$$
Y_{a}^{*}=-\left(1-Y_{1 \infty}\right)\left[\exp \left(m_{0}\right) \frac{L}{c_{p} T_{\infty}}\right]^{-1} \theta_{a}^{*}
$$

and then to calculate corrections to the concentration field.

Equation (32) shows that the vaporization rate coefficent, that is constant in the classical $Q S$ analysis, is corrected due to unsteady effects by a term of order $\sqrt{\bar{\varepsilon}}$ which varies with time. The square of the droplet radius varies no longer linearly with time, equation (33) if the $N S$ effects are retained.

The vaporization time, $\tau_{v}$, is obtained from equation (33) by making $x_{s}$ equal to zero. We thus obtain an explicit relation between $\varepsilon m_{0}$ and $2 m_{0} \tau_{v}$. For small values of $\varepsilon m_{0}$, the error of the $Q S$ analysis that gives:

$$
\tau_{v}=1 / 2 m_{0}
$$

is found to be small. If terms of order $\varepsilon \ln \varepsilon$ are neglected, we obtain from equation (33)

$$
2 m_{0} \tau_{v}=1-\sqrt{2 \varepsilon m_{0} / \pi}
$$

The fact that the droplet life-time, and thus the boundary conditions for the equations, change with the order of approximation indicates that one should use a stretched time variable,

$$
\tau^{\prime}=\tau\left(1+b_{1} \sqrt{\varepsilon}+\ldots\right),
$$

instead of the non-dimensional time $\tau$ that has been used; the coefficients $b_{i}$ should be chosen so that the vaporization time $\tau_{v}^{\prime}$ does not change with higher order approximations. If the analysis presented above is remade in this way, no changes are found in the previous results if they are written in terms of $\tau^{\prime}$, with the exception of equation (33) which is replaced by

$$
\begin{aligned}
x_{s}^{2}= & 1-2 m_{0} \tau^{\prime}\left(1-b_{1} \sqrt{\varepsilon}\right)-\sqrt{2 \varepsilon m_{0} / \pi} \\
& \times\left[\sqrt{2 m_{0} \tau^{\prime}}+\frac{1-2 m_{0} \tau^{\prime}}{2} \ln \frac{1+\sqrt{2 m_{0} \tau^{\prime}}}{1-\sqrt{2 m_{0} \tau^{\prime}}}\right]
\end{aligned}
$$

So that $b_{1}=\sqrt{2 m_{0} / \pi}$ if we want the droplet lifetime to be given by $2 m_{0} \tau_{v}^{\prime}=1$ also in the second approximation. In terms of the variable $\tau$, to this approximation, we obtain for $\tau_{v}$

$$
2 m_{0} \tau_{v}=\left\{1+\sqrt{2 \varepsilon m_{0} / \pi}\right\}^{-1}
$$




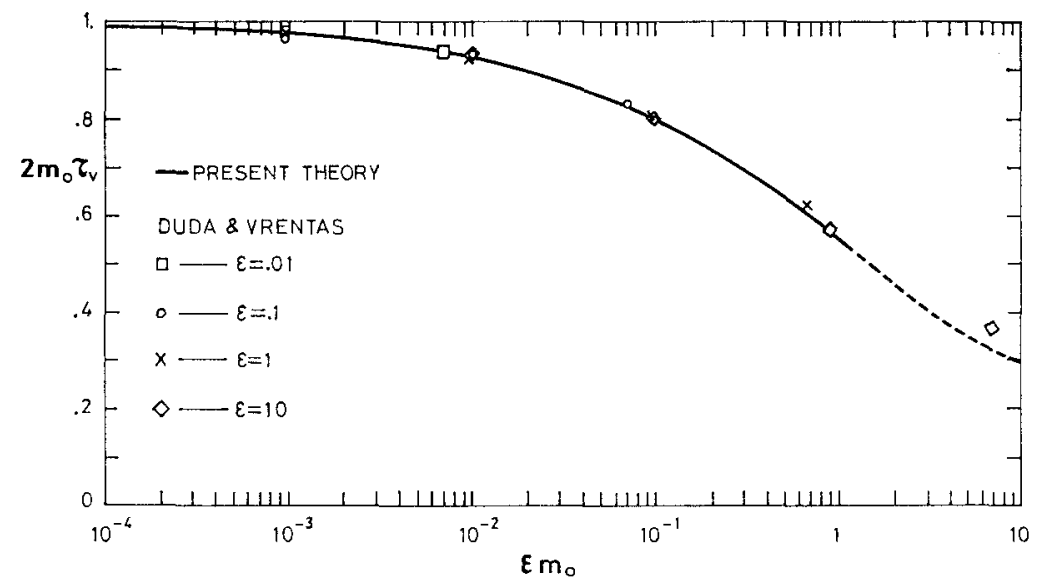

FIG. 2. Vaporization time as a function of $\varepsilon m_{0}$. Comparison with the numerical sphere dissolution lifetime results of Duda and Vrentas (1971), shown by Rosner and Chang (1973) to be applicable to fuel droplet evaporation-combustion under $N S$-conditions.

which has been plotted in Figure 2 and is found to correlate well the numerical results of Duda and Vrentas (1971). It should be remarked that the quantity $2 m_{0} \tau_{v}$ is identical to the dimensionless droplet lifetime $\tau_{\text {life }}$ introduced by Rosner and Chang (1973). It is simply the ratio of the actual droplet lifetime to that formally predicted by the $Q S$-approximation.

Figure 3 gives the deviation from the linear $Q S$ result in the square of the droplet radius as a function of $2 m_{0} \tau^{\prime}=\tau / \tau_{v}$, given by equation (33').

\subsection{Temperature and Concentration Distributions for Times Larger Than the Vaporization Time.}

During the droplet vaporization period, $\tau<\tau_{v}$, the inner region is scaled with $x_{s}$ that vanishes at $\tau=\tau_{v}$. At times $\tau>\tau_{v}$ the temperature and concentration distributions are given by the outer expansion (21), which is now valid everywhere, if the upper limit of integration in equation (24) is replaced by $1 / 2 m_{0}$, because there are no sources of heat and mass at $X=0$ at $\tau>\tau_{v} \simeq 1 / 2 m_{0}$.

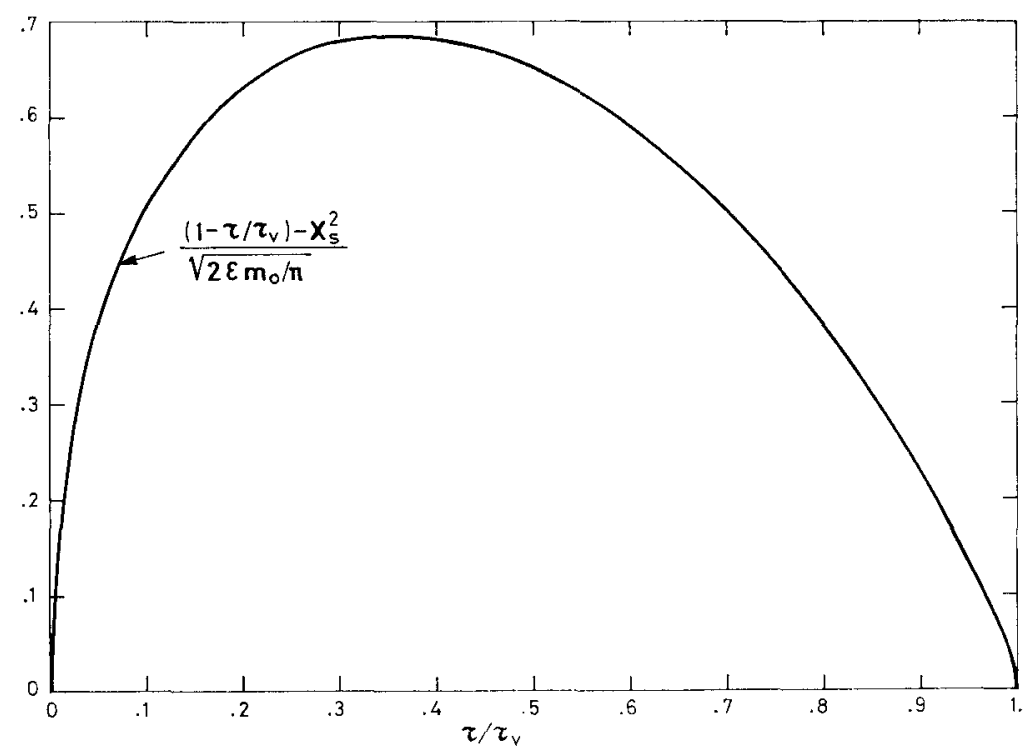

FIG. 3. Deviation from the linear law of variation of $x_{8}^{2}$ with time. 


\section{DROPLET VAPORIZATION WITH COMBUSTION}

In this case the vapor leaving the droplet reacts exothermically with the oxidizer leading to an increase in the heating of the droplet that enhances its vaporization rate. This reaction is represented by production terms that appear in the right hand side of equations (2) and (3). However, the variables

$$
\begin{aligned}
& \bar{Y}=Y_{1}-Y_{2} / \nu \\
& \bar{\theta}=\theta+q_{r} Y_{2} / v c_{p} T_{\infty}
\end{aligned}
$$

satisfy the following equations without the chemical production terms,

$$
\mathscr{L}(\bar{Y})=\mathscr{L}(\bar{\theta})=0
$$

where $v$ and $q_{r}$ are respectively the mass of oxidizer consumed and heat released per unit mass of fuel consumed. The operator $\mathscr{L}$ is defined in formula (4).

If the thin flame assumption is used, we find that inside the flame $Y_{2}$ is equal to zero and, thus, $\bar{Y}=Y_{1}$ and $\bar{\theta}=\theta$, so that $\bar{Y}$ and $\bar{\theta}$ satisfy not only the same equations, (2) and (3), as $Y_{1}$ and $\theta$ in the pure vaporization case, but also the same boundary conditions at the droplet surface, equations $(5)-\left(8^{\prime}\right)$. However, since $Y_{1}=0$ outside the thin flame, the initial and boundary conditions at infinity for $\bar{Y}$ and $\bar{\theta}$ are

$$
\bar{Y}=-Y_{2 \infty} / \nu \quad \bar{\theta}=1+q_{r} Y_{2 \infty} / c_{p} T_{\infty} \nu
$$

instead of the equations (9) and (10) used for $Y_{1}$ and $\theta$ in the pure vaporization case.

The results obtained in the previous section can now be used directly to generate the first and second approximation for $\bar{Y}$ and $\bar{\theta}$ in the inner and outer regions. In particular, the first approximation, $m_{0}$, for the vaporization coefficient, $m$, is given by

$$
m_{0}=\ln \left\{\left(1+q_{r} Y_{2 \infty} / v L\right)+\left(1-\theta_{B}\right) c_{p} T_{\infty} / L\right\}
$$

that can be used to calculate, in the outer region, the first perturbations of $\bar{Y}$ and $\bar{\theta}$ from their values at infinity; these are given by equation (24) together with equations (27) and (28). Notice that the factor $\left(1-Y_{1 \infty}\right)$ in equation (28) must be replaced by $\left(1+Y_{2 \infty} / v\right)$. Then, as in the previous section, we can proceed to calculate the second approximation for $\bar{Y}$ and $\bar{\theta}$, as well as $m$ and $r_{s}$ that are given by equations (32) and (33') together with (41). The second approximation of the vaporization time is given by equations $\left(36^{\prime}\right)$ and (41).
The flame position can be obtained as a function of time by solving the equation $\bar{Y}\left(x_{f}, \tau\right)=0$. The solution will be obtained, first, for the case in which the flame is in the inner region that correspond to $Y_{2 \infty} / v \sim 1$, and, then, for the case in which the flame is in the outer region when $Y_{2 \infty} / v \sim \sqrt{\varepsilon}$.

\subsection{Flame in the Inner Region}

The first two terms of the inner expansion for $\bar{Y}$ can be obtained from the solution of the $Q S$ forms of equation (39) and of the boundary condition (7). Then

$$
\bar{Y}=1-\left(1-\bar{Y}_{a \infty}\right) \exp \left(-m x_{s} \mid x\right)
$$

The apparent concentration at infinity

$$
\bar{Y}_{a \infty}=-Y_{2 \infty} / \nu+\sqrt{\varepsilon} Y_{a}^{*}
$$

includes a term of order $\sqrt{\varepsilon}$ obtained from the matching conditions with the outer expansion According to equation (34).

$$
Y_{r}^{*} /\left(1+Y_{2 \infty} / \nu\right)=-m_{1}
$$

The equation $\bar{Y}=0$ can now be solved, and then the first two terms of an expansion of the ratio $x_{f} / x_{s}$ are obtained.

$$
\begin{aligned}
\frac{x_{f}}{x_{s}}= & \frac{m_{0}}{\ln \left(1+Y_{2 \infty} / v\right)} \\
& \times\left[1+\sqrt{\varepsilon} \frac{m_{1}}{m_{0}}\left(1-\frac{m_{0}}{\ln \left(1+Y_{2 \infty} / v\right)}\right)\right]
\end{aligned}
$$

\subsection{Flame in the Outer Region}

The first and second terms of the expansion for $\left(x_{f} / x_{s}\right)$, given by equation (45), become of the same order, $1 / \sqrt{\varepsilon}$, when $Y_{2 \infty} / \nu m_{0} \sim \sqrt{\varepsilon}$. This occurs when the oxidizer concentration at infinity is low or the stoichiometric ratio is high; then the flame has to go far from the droplet, to the outer region, so that fuel and oxidizer will arrive to the flame in the stoichiometric ratio.

The flame position in this case has to be found by solving the equation

$$
\begin{aligned}
\bar{Y}= & -Y_{2 \infty} / v+\frac{\sqrt{\varepsilon} m_{0}\left(1+Y_{2 \infty} / v\right)}{2 \sqrt{\pi}} \\
& \times \int_{0}^{\gamma} \frac{\sqrt{1-2 m_{0} \xi}}{(\tau-\xi)^{3 / 2}} e^{-X_{f}^{2 / 4(\tau-\xi)}} d \xi=0
\end{aligned}
$$

where $\gamma=\tau$ if $2 m_{0} \tau<1$, and $2 m_{0} \gamma=1$ if $2 m_{0} \tau>1$. In this case $Y_{2 \infty} / \nu$ is small, so that the factor $\left(1+Y_{2 \infty} / \nu\right)$ will be left out of equation (46). 
Notice that when the droplet disappears at $\tau=\tau_{v}$, the flame radius does not become zero and takes the value given by the equation

$$
E_{1}\left(m_{0} X_{f}^{2} / 2\right)=\left(2 \pi / m_{0}^{3} \varepsilon\right)^{1 / 2} Y_{2 \infty} / \nu=\alpha
$$

obtained from (46) by setting $\gamma=\tau=\tau_{v}=1 / 2 m_{0}$, where $E_{1}$ is the exponential integral function. For large values of the parameter $\alpha, X_{f}$ becomes very small; this means that in this limit, corresponding to the flame in the inner region, the flame and droplet radius become zero simultaneously.

The extinction time, $\tau_{e}$, obtained by setting $X_{f}=0$ in equation (46), is given by the relation

$$
\alpha=-\frac{2}{\sqrt{\tau_{e} / \tau_{v}}}+\ln \frac{\sqrt{\tau_{e} / \tau_{v}}+1}{\sqrt{\tau_{e} / \tau_{v}}-1}
$$

For small values of $\alpha, \tau_{e} / \tau_{v} \gg 1$. Then, during most of the flame lifetime $\tau \gg \tau_{v}>\xi$ and the integral in equation (46) can be simplified

$$
m_{0} X_{f}^{2} / 2=\left(\tau / \tau_{v}\right) \ln \left\{(2 / 3 \alpha)\left(\tau / \tau_{v}\right)^{-3 / 2}\right\}
$$

The same result was obtained by Spalding (1959) considering the droplet as an instantaneous source of vapor.

In Figure 4 the flame radius multiplied by $\sqrt{m_{0} / 2}$, as function of the ratio $\tau / \tau_{v}$, is represented for different values of the parameter $\alpha$, as obtained from equation (46). Figure 4 includes a comparison with the $Q S$ theory for a large value, 10, of $\alpha$, and with Spalding's result for a small value, 0.05 , of $\alpha$.

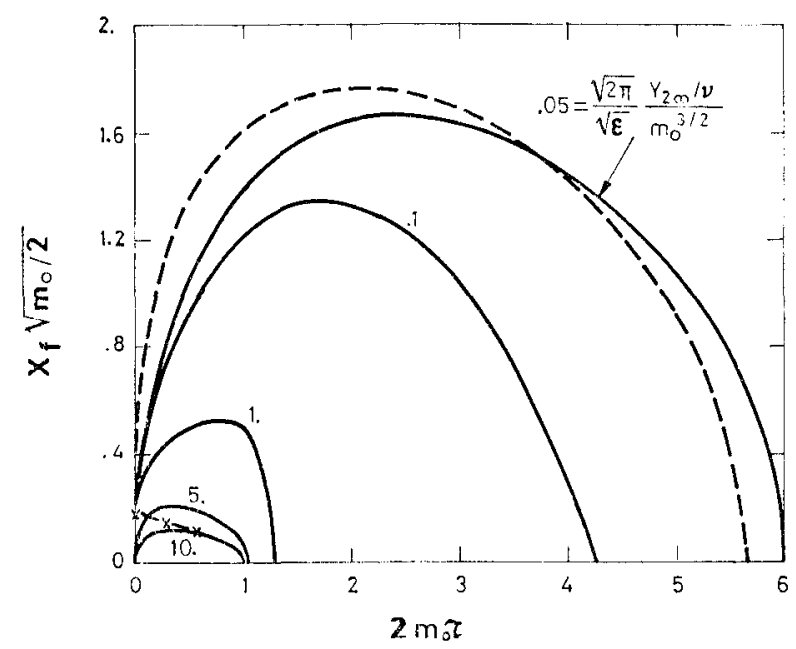

FIG. 4. Flame radius as a function of time, and comparison with Spalding (1959) source solution for $\alpha=0.05,----$, and with the $Q S$ solution for $\alpha=10,-x-x-$

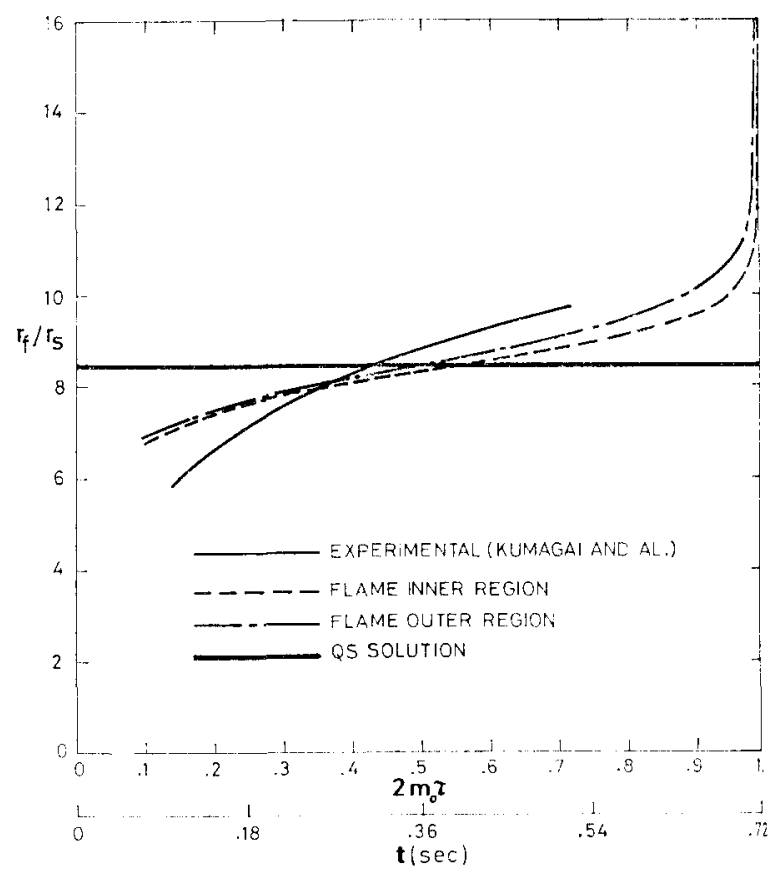

FIG. 5. Ratio of flame to droplet radius as a function of time. Comparison of the present results, equations (45) and (46), with the experimental results of Kumagai et al. (1971).

As it was pointed out in the Introduction, the experimental results on the combustion of single droplets show that the " $d^{2}$ law" of burning is valid, but the ratio of flame to droplet diameter changes during combustion, whereas the $Q S$ theory will predict that it is constant, The results shown above suggest that this may be due to the fact that the flame is located far from the droplet. For example for $n$-heptane burning in air $Y_{2 \infty} / v=0.065$, and the value of $\varepsilon$, for a temperature at infinity of $1200^{\circ} \mathrm{K}$, is $\varepsilon=0.42 \times 10^{-3}$. Consequently, in a situation like this, $\sqrt{\varepsilon}$ and $Y_{2 \infty} / v$ are of the same order, and the flame could be located in the outer $N S$ region. While the expected errors in using the classical $Q S$ theory for vaporization are of order $\sqrt{\varepsilon}$, the relative errors will be of order unity in calculating the flame position.

In Figure 5 a comparison is given of the experimental results of Kumagai et al. (1971) and our theoretical calculations. The conditions under which the experiments of Kumagai et al. have been carried out are such that the flame is in the zone of common validity of the outer (46) and inner (45) solutions, and for this reason both of them have been drawn in Figure 5. 
Regarding this point, it should be mentioned that if the flame would have been located clearly in the outer region, the theory here presented should not be applicable to this experiment, because the heat of reaction would be so large as to make the temperature of the flame considerably larger than the temperature at infinity, thus invalidating the assumption $\theta=1+\sqrt{\varepsilon} \theta^{*}$ that allows to linearize the equations in the outer region.

\section{CONCLUDING REMARKS}

Unsteady effects in droplet vaporization are due on one hand to the thermal inertia of the liquid, so that an initial period exists in which the liquid temperature rises from its initial value to a temperature close to the boiling temperature; for moderately large values of the nondimensional heat of vaporization $L / R_{1} T_{B}$ no significant vaporization occurs during this heat up period and $T=T_{B}$ afterwards. The analysis presented here does not consider the first heat-up period, and it refers to the main period of vaporization, when the liquid temperature is constant, and the unsteady effects are represented by the time derivative terms in the gas phase equations. We have found that in an inner region, $r \sim r_{s}$, these terms become of order $\varepsilon$ relative to the convective or diffusive terms after a short time period of adjustment of the temperature and concentration distributions to their quasisteady forms. This period of time is of order $\varepsilon$ relative to the droplet lifetime. We have not taken into account the existence of this initial stage because it leads to changes in the vaporization rate and droplet lifetime of order $\varepsilon$.

For the droplet vaporization time we have found the value

$$
t_{v}=\left(r_{s o}^{2} \rho_{l} c_{p} / 2 \lambda m_{0}\right)\left\{1+\sqrt{2 m_{0} \rho_{\infty} / \rho_{l} \pi}\right\}^{-1}
$$

where $m_{0}$ is given by equation (17) for the pure vaporization case, and by equation (41) for the case of vaporization enhanced by combustion. The factor in curly brackets accounts for the unsteady effects. They are dependent on the parameter $\varepsilon m_{0}$ which should have been used instead of $\varepsilon$ as the small parameter in the asymptotic analysis. Equation $(50)$ is found to correlate very well, even for values of $\varepsilon m_{0}$ of order unity, the results obtained by Duda and Vrentas (1971) by numerical integration of the unsteady differential equations for the problems of the dissolution of a sphere. Indeed, provided $\rho_{\infty} / \rho_{l}$ is sufficiently small equation (50) can now be safely exploited to extend useful crossplots of Duda and Vrentas' numerical results (of Rosner and Chang (1973)) to values of the driving parameter $m_{0}$ in the range of combustion interest $\left(m_{0}>\right.$ $\ln 2)$.

This research has been sponsered in part by U.S. Air Force Office of Scientific Research under Grants AFOSR-72-2253 and AFOSR-73-2535. We thank T. C. Adamson for helpful discussions.

\section{REFERENCES}

Chervinsky, A. (1969). Transient burning of spherica symmetric fuel droplets. Israel J. Tech 7, 66-73.

Duda, J. L. and Vrentas, J. S. (1971). Heat or mass transfer controlled dissolution of an isolated sphere. Int.J. Heat Mass Transfer 14, 395.

Isoda, H. and Kumagai S. (1958). New aspects of droplet combustion. Seventh Symposium (International) on Combustion. Butterworths, p. 523.

Kaplun, S. and Lagerstrom, P. A. (1957). Asymptotic expansions of Navier-Stokes solutions for small Reynolds numbers. J. Math. Mech. 6, 595.

Kotake, S. and Okazaki, T. (1969). Evaporation and combustion of a liquid droplet. International Journal of Heat and Mass Transfer 12, 595.

Krier, H. and Wronkiewcz, J. A. (1972). Combustion of single drops of fuel. Combustion and Flame 18, 159.

Kumagai, S., Sakai, T., and Okajima, S. (1971). Combustion of free fuel droplets in a freely falling chamber. 13 th Symposium (International) on Combustion. The Combustion Institute, p. 779.

Manrique, J. A. and Borman, G. L. (1969). Calculation of steady state droplet vaporization at high ambient pressures. Int. J. Heat Mass Transfer 12, 1081.

Matlosz, R. L., Leipziger, S., and Torda, J. P. (1972). Investigation of liquid drop evaporation in a high temperature and high pressure environment. Int. J. Heat.Mass Transfer 15, 831 .

Nuruzzaman A. S. M. and Beer J. M. (1971). On the nonsteady nature of droplet combustion. Combustion Science and Technology 3, 17

Rosner, D. E. (1967). On liquid droplet combustion at high pressures. AIAAJ. 5, 163.

Rosner, D. E. (1972). Liquid droplet vaporization and combustion. Liquid propellant rocket combustion instability. NASA Scientific and Technical Information Office, Chap. 2.4, 74-100, NASA SP-194.

Rosner, D. E. and Chang W. S. (1973). Transient evaporization and combustion of a fuel droplet near its critical temperature. Combustion Science and Technology 7,145.

Rush J. H. and Krier H. (1973). Burning of fuel droplets at elevated pressures. University of Illinois Technica Report AAE 73-8, UILA-Eng 730508.

Spalding, D. B. (1959). Theory of particle combustion at high pressures. ARS Journal 29, 828.

Tarifa, C. and Crespo A. (1972). Droplet combustion at high pressures with unsteady effects. Air Force Office of Scientific Research, Office of Aerospace Research. Grant F61052-69-C-0035. Final Report. 
Tarifa, C., Crespo, A., and Fraga, E. (1972). A theoretical model for the combustion of droplets and gas pockets in supercritical conditions. Astronautica Acta 17, 685.

Wieber, P. R. (1963). Calculated temperature histories of vaporizing droplets to the critical point. $A I A A J .1$, 2764.
Williams, F. A. (1959). On the assumptions underlying droplet vaporization and combustion theories. Lockhed AFOSR TN 59-1148; also J. Chem. Phys. 33, 133 (1960). Williams, A. (1973). Combustion of droplets of liquid fuels: a review. Combustion and Flame 21, 1.

Revised ms. received September 30, 1974 